\title{
Histologic Assessment of Non-Small Cell Lung Carcinoma after Neoadjuvant Therapy
}

Xiaolin Liu-Jarin, M.D., Ph.D., Mark B. Stoopler, M.D., Haralambos Raftopoulos, M.D., Mark Ginsburg, M.D., Lyall Gorenstein, M.D., Alain C. Borczuk, M.D.

Departments of Pathology (XL-J, ACB), Medicine (MBS, HR), and Surgery (MG, LG), Columbia

Presbyterian Medical Center, New York, New York

Chemotherapy or chemoradiation is often used in Stage IIIA non-small cell lung carcinoma before surgical resection (neoadjuvant therapy). In reviewing the histopathology of such tumors after resection, the recognition that the pathologic changes are related to prior therapy and the assessment of tumor regression are both of importance. To refine histologic parameters for tumor regression and describe patterns of tumor reaction to therapy, we identified 30 lobectomy or pneumonectomy specimens from 1996-2000 in which neoadjuvant therapy was received before surgical resection. Histologic patterns of treatment-induced tumor regression were analyzed semiquantitatively and included necrosis, fibrosis, mixed inflammatory infiltrate, foamy macrophages, and giant cells. To identify clinical and histologic parameters that correlate with treatment response, the 30 specimens were graded for tumor regression. No correlation was found between tumor regression and age, gender, or type of therapy (chemoradiation versus chemotherapy alone). Squamous cell carcinoma showed a significantly higher rate of response than adenocarcinoma $(P$ $=.04)$, with a significant number of adenocarcinomas in the nonresponder subgroup $(P=$ .05). Tumor size reduction by radiologic assessment, when compared with histologic regression, did not reveal a statistically significant association. However, a positive correlation was found between extent of fibrosis and radiologic estimate of size reduction.

Copyright () 2003 by The United States and Canadian Academy of Pathology, Inc.

VOL. 16, NO. 11, P. 1102, 2003 Printed in the U.S.A.

Date of acceptance: August 15, 2003.

Address reprint requests to: Alain C. Borczuk, M.D., Columbia University, College of Physicians and Surgeons, Dept. of Pathology VC 14-215, 630 West 168th Street, New York, NY 10032; fax: 212-305-2301; e-mail: ab748@columbia.edu.

DOI: 10.1097/01.MP.0000096041.13859.AB
KEY WORDS: Lung carcinoma, Pathology, Neoadjuvant, Non-small cell, Response.

Mod Pathol 2003;16(11):1102-1108

Non-small cell carcinoma of the lung represents a histologically diverse group of tumors that are broadly classified into three groups: namely, adenocarcinoma, squamous carcinoma, and large cell carcinoma (1). In the last decade, it has been suggested that the use of neoadjuvant therapy (chemotherapy or chemoradiation), followed by surgical resection, in locally advanced disease improves survival in this subgroup (2-7). In fact, the prognosis of patients in whom a pathologic complete response is documented is dramatically improved $(8,9)$. The beneficial effects of induction chemotherapy for patients with earlier stage lung cancer $(9,10)$ remain to be fully explored.

These developments in non-small cell carcinoma have direct implications for the pathologic assessment of resected tumors. Histologic recognition of cases in which neoadjuvant therapy has been given and the description of extent of residual tumor will become increasingly important in prognostication and in evaluating postoperative therapeutic options. Although definitions of pathologic complete response are clear (no residual tumor), complete responders represent the minority of cases, and the benefit of therapy may extend to some patients with a small amount of residual tumor $(8,9)$. The significance of partial response is, however, less clear. The prior studies of Junker et al. $(11,12)$ began the process of clarifying clinically relevant tumor regression categories. In our series, pathologic features seen in tumor regression and their relationship to clinical parameters in patients receiving neoadjuvant therapy will be emphasized.

\section{MATERIALS AND METHODS}

Using a natural language search of all resected lung carcinoma from 1996-2000, we identified 30 patients with non-small cell lung carcinoma who 
received neoadjuvant therapy before definitive surgery during this time period. Histologic slides on all 30 cases were reviewed. In 22 cases, biopsy materials before therapy were available for direct histologic comparison. A combination of biopsy or mediastinal node sampling and assessment of residual tumor led to classification as adenocarcinoma or squamous carcinoma.

In the lobectomy and pneumonectomy specimens, sections taken from the residual mass were reviewed. In cases with no residual carcinoma, the entire tumoral mass was submitted. Histologic parameters were analyzed, including tumor necrosis, foam cell reaction, giant cell reaction, inflammation, and fibrosis. These parameters were chosen based on comparison between pretreatment biopsy and resection. These changes were semiquantitatively graded as 0 (not present), 1 (focally present), 2 (moderately present), or 3 (extensively present).

Tumors were divided into different response groups according to the volume of residual tumor. The percentage of residual tumor was estimated by comparing the estimated cross-sectional area of the largest residual tumor focus to the estimated crosssectional area of the inflammatory or fibrous mass. The regression response was classified using a modification of the method of Junker et al. (12). Given the nature of their results, we re-named their four categories (3 groups) using the same numerical guidelines but reflecting only two groups-Group I as nonresponders and Group II as responders, each with two subgroups to maintain the Junker et al. (12) classification scheme. Therefore we divided our cases into the following: Grade Ia, marginal or no regression; Grade $\mathrm{Ib}$, morphologic evidence of therapy-induced changes but $>10 \%$ residual tumor; Grade IIa, extensive response but with residual tumor $\leq 10 \%$; and Grade IIb, pathologic complete response.

Information regarding the total dose of radiation therapy and the interval between last radiation treatment and surgery was obtained in 14 of 20 patients who received radiation. The average dose of radiation was $4970 \pm 260 \mathrm{cGy}$ (with 13 of the 14 receiving $5040 \mathrm{cGy}$ ), and the average interval between last radiation dose and surgery was $6.1 \pm 0.9$ weeks.

Tumor regression was correlated with clinical parameters including patient age, gender, $\mathrm{T}$ status, $\mathrm{N}$ status, type of therapy, radiologic estimate of size reduction, and the previously stated histologic parameters. Radiologic estimates of tumor size reduction were based on pre- and posttreatment CAT scan reports: we used either the change in two perpendicular dimensions or direct statements as to the percentage change that were made within reports comparing studies. If neither of these methods was possible, the data were not included, re- sulting in exclusion of five patients from the statistical analysis.

Tumors were staged using the TNM staging system (13). Preoperative staging was based on radiologic findings and tissue sampled at mediastinoscopy. Postoperative staging involved pathologic assessment of residual tumor. Three patients were M1. Two patients had a solitary brain metastasis, and one patient had a solitary adrenal metastasis. For survival analysis, only patients with a minimum of 6 months' postoperative survival were included. Two patients were lost to follow-up. Survival times were calculated from date of surgery up to June 2001. Univariate analysis of survival and response group was performed with Fisher's exact test and a threshold of significance of $P=.05$; survival times were compared with those of response groups using the log rank test.

\section{RESULTS}

A total of 30 cases was examined and classified as to the extent of tumor response. Tumor response was divided into two groups (I, nonresponder and II, responder), each of which contained two subgroups ( $\mathrm{a}$ and $\mathrm{b})$. Of the nonresponder group ( $n=$ 17), 6 patients had little to no evidence of response (Subgroup Ia), and 11 patients had identifiable features associated with response but had $>10 \%$ residual tumor (Subgroup Ib). Of the responder group ( $n=13), 8$ cases had extensive response (Subgroup IIa), and 5 cases had pathologic complete response (Subgroup IIb). No cases in our series were difficult to classify based on a $10 \%$ cutoff, as most cases were either well below or well above that number (Table 1)

There was no correlation between $\mathrm{T}$ status and $\mathrm{N}$ status and likelihood of response. Examination of pre- and posttreatment staging showed that overall nodal response mirrored tumor response. Of the node-positive patients in the responder group, 8 of $10(80 \%)$ were $\mathrm{N}_{0}$ after therapy, whereas in the nonresponder group, only 3 of 14 preoperative node-positive patients were $\mathrm{N}_{0}$ after therapy.

The histopathologic classification of the tumors was examined. In the responder group, 8 of 13 cases were squamous carcinomas, whereas 5 of 13 were adenocarcinomas. In the nonresponder group, 4 of 17 patients had squamous carcinomas, and 13 of 17 patients had adenocarcinomas (Table 1). Squamous carcinoma was associated with a higher probability of treatment response $(P=.04$, Fisher's exact test). In addition, all cases in Subgroup Ia were adenocarcinomas, and this was also statistically significant ( $P=.05$, Fisher's exact test).

Figure 1 shows typical examples of the histopathology of tumors associated with complete or ex- 
TABLE 1. Patient Demographics, Treatment Information, and Tumor Regression

\begin{tabular}{|c|c|c|c|c|c|c|c|c|c|c|}
\hline & $\begin{array}{l}\text { Residual } \\
\text { tumor } \\
(\%)\end{array}$ & $\begin{array}{l}\text { Regression } \\
\text { Group }\end{array}$ & Age & Gender & $\begin{array}{c}\text { Preoperative } \\
\text { TNM }\end{array}$ & $\begin{array}{c}\text { Preoperative } \\
\text { Stage }\end{array}$ & $\begin{array}{c}\text { Postoperative } \\
\text { TNM }\end{array}$ & Histology & Therapy & $\begin{array}{c}\text { Survival/ } \\
\text { Survival } \\
\text { Time (Mo) }\end{array}$ \\
\hline \multirow[t]{17}{*}{ Nonresponder } & 100 & Ia & 45 & $\mathrm{~F}$ & T3N0M1 & IV & T3N0M1 & $\mathrm{AD}$ & Chemo (CisP, V) & Expired, 9 \\
\hline & 95 & Ia & 65 & $\mathrm{~F}$ & T3N2M0 & IIIA & T2N0M0 & $\mathrm{AD}$ & Chemo $(\mathrm{P}, \mathrm{Cis} \mathrm{P})+\mathrm{RT}$ & Expired, 17 \\
\hline & 95 & Ia & 75 & M & T2N2M0 & IIIA & T3N1M0 & $\mathrm{AD}$ & Chemo (P, CarboP) & Expired, 7 \\
\hline & 95 & Ia & 60 & M & T3N2M0 & IIIA & T3N2M0 & $\mathrm{AD}$ & Chemo (P, CarboP) & Alive, 26 \\
\hline & 90 & Ia & 54 & M & T2N2M0 & IIIA & T2N2M0 & $\mathrm{AD}$ & Chemo + RT & Expired, 11 \\
\hline & 90 & Ia & 53 & M & T3N0M1 & IV & T3N0M1 & $\mathrm{AD}$ & Chemo + RT & LTF \\
\hline & 80 & $\mathrm{Ib}$ & 62 & M & T2N2M1 & IV & $\mathrm{T} 2 \mathrm{~N} 2 \mathrm{M} 1$ & $\mathrm{AD}$ & Chemo $(\mathrm{P}$, CarboP $)+\mathrm{RT}$ & Alive, 17 \\
\hline & 50 & $\mathrm{Ib}$ & 73 & M & T3N1M0 & IIIA & T2N1M0 & SQ & Chemo & LTF \\
\hline & 50 & $\mathrm{Ib}$ & 54 & M & T3N2M0 & IIIA & T3N1M0 & SQ & Chemo + RT & Alive, 15 \\
\hline & 40 & $\mathrm{Ib}$ & 73 & M & T3N0M0 & IIB & T3N0M0 & $\mathrm{AD}$ & Chemo (V, CisP) + RT & Expired, 2 \\
\hline & 40 & $\mathrm{Ib}$ & 56 & M & T4N2M0 & IIIB & T3N2M0 & $\mathrm{AD}$ & Chemo $(\mathrm{M}, \mathrm{CisP})+\mathrm{RT}$ & Expired, 14 \\
\hline & 40 & $\mathrm{Ib}$ & 71 & M & T2N2M0 & IIIA & T2N0M0 & SQ & Chemo + RT & Expired, 1 \\
\hline & 40 & $\mathrm{Ib}$ & 73 & $\mathrm{~F}$ & T2N2M0 & IIIA & T2N2M0 & SQ & Chemo (CisP, P) & Expired, 33 \\
\hline & 40 & $\mathrm{Ib}$ & 41 & M & T1N2M0 & IIIA & T1N0M0 & $\mathrm{AD}$ & Chemo (CisP, V) + RT & Expired, 25 \\
\hline & 40 & $\mathrm{Ib}$ & 51 & M & T2N2M0 & IIIA & T2N1M0 & $\mathrm{AD}$ & Chemo (CisP, V) + RT & Alive, 34 \\
\hline & 40 & $\mathrm{Ib}$ & 67 & $\mathrm{~F}$ & T2N2M0 & IIIA & $\mathrm{T} 2 \mathrm{~N} 2 \mathrm{M} 0$ & $\mathrm{AD}$ & Chemo $($ CisP, $\mathrm{V})+\mathrm{RT}$ & Alive, 39 \\
\hline & 20 & $\mathrm{Ib}$ & 65 & M & T2N2M0 & IIIA & T2N2M0 & $\mathrm{AD}$ & Chemo $(\mathrm{P}$, CarboP) $+\mathrm{RT}$ & Expired, 4 \\
\hline \multicolumn{11}{|l|}{ Responders } \\
\hline & 5 & IIa & 39 & $\mathrm{~F}$ & T3N1M1 & IV & T1N0M0 & SQ & Chemo (Velban, CisP, M) + RT & Alive, 67 \\
\hline & 5 & IIa & 52 & M & T1N2M0 & IIIA & T1N0M0 & SQ & Chemo (Velban, CisP, M) + RT & Alive, 59 \\
\hline & 5 & IIa & 61 & $\mathrm{~F}$ & T2N2M0 & IIIA & T1N0M0 & SQ & Chemo (CisP, P) & Expired, 1 \\
\hline & 5 & IIa & 65 & $\mathrm{~F}$ & T3N2M0 & IIIA & T3N2M0 & $\mathrm{AD}$ & Chemo $(\mathrm{P})+\mathrm{RT}$ & Alive, 7 \\
\hline & 5 & IIa & 65 & M & T1N2M0 & IIIA & T1N0M0 & $\mathrm{AD}$ & Chemo $(\mathrm{V}, \mathrm{CisP})+\mathrm{RT}$ & Expired, 8 \\
\hline & 5 & IIa & 49 & $\mathrm{~F}$ & T3N2M0 & IIIA & T3N2M0 & SQ & Chemo (P, CarboP, MMPI) & Alive, 25 \\
\hline & 5 & IIa & 69 & M & T3N0M0 & IIB & T3N0M0 & $\mathrm{AD}$ & Chemo $(\mathrm{V}, \mathrm{CisP})+\mathrm{RT}$ & Expired, 10 \\
\hline & 5 & IIa & 71 & M & T2N1M0 & IIB & T2N0M0 & SQ & Chemo (P,CisP) & Alive, 42 \\
\hline & 0 & IIb & 51 & $\mathrm{~F}$ & T3N0M0 & IIB & TONOM0 & SQ & Chemo + RT & Expired, 1 \\
\hline & 0 & $\mathrm{IIb}$ & 69 & M & T3N0M0 & IIB & TONOM0 & SQ & Chemo (P, CarboP) $+\mathrm{RT}$ & Alive, 19 \\
\hline & 0 & $\mathrm{IIb}$ & 66 & M & T2N3M0 & IIIB & TONOM0 & SQ & Chemo (P, CarboP) & Alive, 22 \\
\hline & 0 & $\mathrm{IIb}$ & 76 & $\mathrm{~F}$ & T3N1M0 & IIIA & TONOM0 & $\mathrm{AD}$ & Chemo $(\mathrm{P}$, CarboP) $+\mathrm{RT}$ & Alive, 32 \\
\hline & 0 & $\mathrm{IIb}$ & 60 & $\mathrm{~F}$ & T3N2M0 & IIIA & TONOM0 & $\mathrm{AD}$ & Chemo (P, CarboP) & Alive, 39 \\
\hline
\end{tabular}

P, paclitaxel; V, vinorelbine; M, mitomycin; CarboP, carboplatin; CisP, cisplatin; RT, radiation therapy; AD, adenocarcinoma; SQ, squamous carcinoma.

tensive response to treatment. In some cases, central "infarct-like" coagulative necrosis was present, often with a rim of histiocytic and lymphocytic reaction at the edge of the necrosis (Fig. 1A-B). In zones at the edge of the necrosis were cells with foamy cytoplasm, likely containing lipid (foamy macrophages). These areas of foamy macrophages were in some instances associated with multinucleated giant cells with cholesterol clefts (Fig. 1C). In most tumors, fibrosis was present; in several cases fibrosis was the predominant manifestation of treatment response (Fig. 1D).

Comparison of tumor histology before and after therapy did not show significant change in subtype or pattern of growth (Fig. 2). In the responder subgroup in which residual tumor was present (Subgroup IIa), the growth pattern and differentiation were similar before and after neoadjuvant therapy, although an increase in nuclear pleomorphism was observed (Fig. 2A-B and Fig. 2C-D, each pair before and after therapy). In cases without significant response (Subgroup Ia), treatment effects were often not recognizable histopathologically (Fig. 2E-F, before and after therapy). In three cases, a significant proportion of residual tumor had a bronchioloalveolar pattern after therapy, although a highergrade invasive adenocarcinoma was present before therapy and also was seen as a component of the residual tumor.
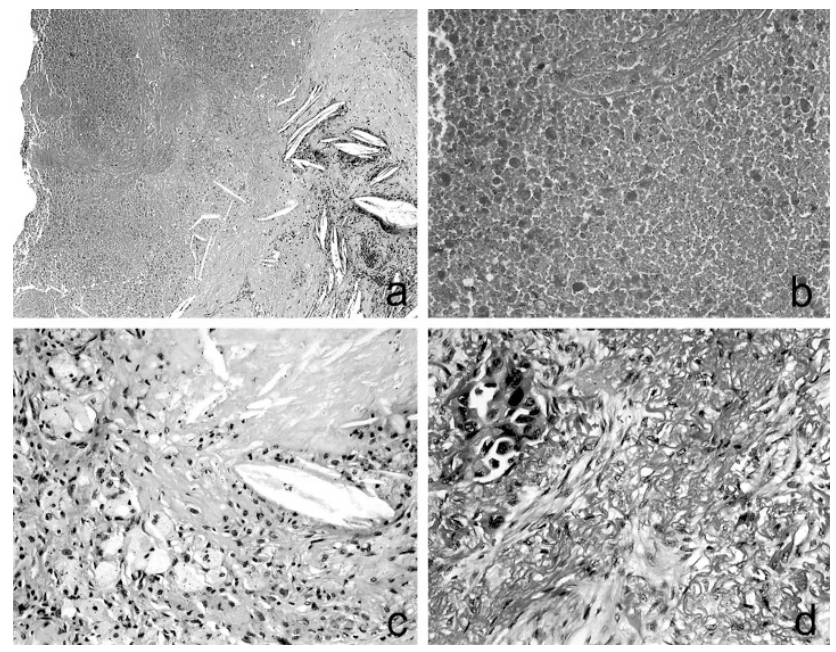

FIGURE 1. Histologic changes seen in pathologic responders. A, a circumscribed area of necrosis is seen with a rim of fibrosis, chronic inflammatory infiltrate, and prominent cholesterol clefts. Such a pattern would have been recorded as $3+$ necrosis with minimal $(1+)$ fibrosis. B, a higher magnification of the central zone shows extensive coagulative necrosis. C, an area adjacent to the necrosis shows "foamy" macrophages and cholesterol clefts. $\mathbf{D}$, in this case, a paucicellular nodule is replaced by fibrosis. A focus of residual carcinoma is seen in the upper left-hand corner (hematoxylin and eosin, original magnification: A, $25 \times$; B-D, $100 \times$ )

Histopathologic changes due to therapy were also examined. Comparison of pretreatment biopsy to resected tumor identified parameters that ap- 


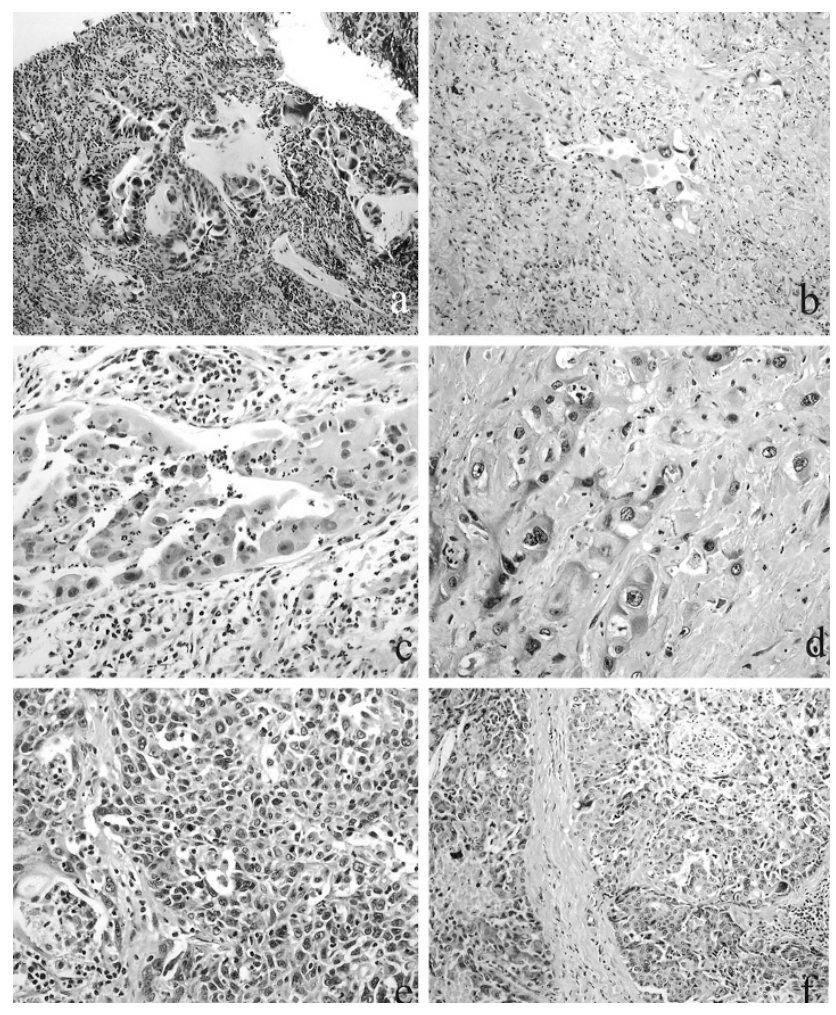

FIGURE 2. Changes in tumor histopathology after neoadjuvant therapy. Each pairing represents the same tumor before and after neoadjuvant therapy. A, pretherapy histology of an adenocarcinoma with moderate nuclear pleomorphism and lymphocytic reaction. B, the same tumor as A after neoadjuvant therapy. Tumor cells show some increase in nuclear pleomorphism and are embedded in fibrotic stroma. C, a moderately differentiated, partly solid growing adenocarcinoma with abundant cytoplasm, before neoadjuvant therapy. $\mathbf{D}$, the same tumor as in $\mathbf{C}$ is seen after therapy amid fibrosis, again with some increase in nuclear pleomorphism. E, a poorly differentiated adenocarcinoma before neoadjuvant therapy. F, the same tumor as in $\mathbf{E}$ after neoadjuvant therapy, showing virtually no change in histology. In F, cholesterol clefts are focally present in the upper left-hand corner. (Hematoxylin and eosin, original magnification: A-B, 20×; C-E, 50×; F, $20 \times)$.

peared to be related to therapeutic effects. Some of these parameters, such as giant cell reaction, were not seen in pretreatment biopsies. Other parameters differed in degree. For example, although apoptosis and focal necrosis with neutrophils was frequently present in pretreatment biopsy, large zones of infarct-like coagulative necrosis were not. This led to a semiquantitative approach to characterize these parameters

Estimates of radiologic tumor response were paired with pathologic regression groupings. The average radiologic size reduction was similar in all subgroups (Table 2). In the responder group, the average regression estimate was $25 \%$, and this was identical in the nonresponder group, using the size reduction criterion of the World Health Organization. No complete responders would have been predicted. A reduction of $>50 \%$ of the sum of two perpendicular dimensions (partial response) was met in only five cases in this study, and one Subgroup Ia and one Subgroup Ib case (pathologic nonresponders) group would have been predicted as partial responders based on this criterion. Also, two of three pathologic complete-response patients (Subgroup IIb) in whom data were available would have been predicted for no change. Pairings of radiologic size reduction with pathologic assessment of response showed no significant correlations (Spearman correlation $r=-.035$ ).

Assessment of the common patterns of tumor response was attempted semiquantitatively, by scoring on a $0-3$ scale. The average values for extent of fibrosis increased with observed extent of response. Interestingly, pairings of estimated radiologic response with extent of fibrosis in patients with $\geq 10 \%$ response showed correlation between estimated radiologic change and extent of fibrosis (Spearman correlation, $r=.46 ; P=.036$ ). Otherwise, the pattern of reaction to therapy did not correlate with other parameters, including initial histology and treatment type.

When examined by the type of therapy received (chemotherapy alone versus chemoradiation), a comparable proportion of patients who received chemotherapy alone $(5 / 10,50 \%)$ and who received chemoradiation $(8 / 20,40 \%)$ were in Group II (responders). Survival data was examined, and an attempt made to correlate subgroups with survival (Table 1). In this small sample, comparison of pathologic complete responders (Subgroup IIb) to the other groups (singly and combined) did not demonstrate a significant difference for survival or survival time. This was examined for Stage IIIA patients as well as for all patients who were Stage IIIA or higher. A comparison of the responder group to the nonresponder group among Stage IIIA patients showed a trend towards survival, although these results did not achieve statistical significance $(P=$ .06 , Fisher's exact test).

TABLE 2. Regression Class, Histologic Features of Tumor Response, and Radiologic Response

\begin{tabular}{lcccc}
\hline $\begin{array}{c}\text { Regression } \\
\text { Class }\end{array}$ & Necrosis* & Fibrosis & $\begin{array}{c}\text { Foam Cell/ } \\
\text { Giant Cells }\end{array}$ & $\begin{array}{c}\text { Radiologic } \\
\text { Response }(\%)\end{array}$ \\
\hline Ia & .33 & .83 & .17 & .33 \\
Ib & 1.7 & 1.9 & .9 & .8 \\
IIa & 1.25 & 2.5 & .375 & .875 \\
IIb & 1.2 & 2.4 & 1.8 & .8 \\
\hline
\end{tabular}

* For necrosis, fibrosis, foam cells/giant cells and inflammation, an average of the semiquantitative score is reported for each subgroup. 


\section{DISCUSSION}

Histologic assessment of tumor regression has been done systematically in a variety of tumors, including osteosarcoma $(14,15)$, head and neck carcinomas (16), esophageal carcinoma (17), and small cell lung carcinoma (18). Although the emphasis of these studies varied, pathologic examination of posttherapy tumor included attempts at classifying tumor response. In the case of esophageal carcinoma (17), pathologic complete response and extensive response with microscopic residual tumor were groups with survival advantage. Therefore, delineation of treatment response groups is also likely to be of interest in non-small cell lung carcinoma.

In non-small cell lung carcinoma, Junker et al. $(11,12)$ have proposed and implemented a classification scheme. In their analysis, three groups were created with two subgroups, for a total of four categories. Using this analysis, they found that not only complete responders (their Group III) but also extensive responders with $<10 \%$ residual tumor (their Group IIb) had an associated improved survival time. They also reported no correlation with presurgical assessment and extent of pathologic tumor regression.

We examined the histologic changes in 30 nonsmall cell lung carcinomas after neoadjuvant therapy. The common histologic features of tumor regression were coagulative necrosis, fibrosis, foam cell/giant cell reaction, as well as mixed inflammatory infiltrate. Although increasing amounts of fibrosis were seen with greater extent of tumor regression, a pattern of response that was predominantly fibrosis versus necrosis did not correlate with extent of response, histologic type, or treatment modality used (chemotherapy or chemoradiation).

Our series is also distinct from that of Junker et al. (12) in that 10 patients received chemotherapy without radiotherapy as part of their neoadjuvant treatment. Focusing on parameters related to tumor alone, these 10 patients could not be separated from those who received chemoradiation based on histopathologic assessment of residual tumor histology, fibrosis, necrosis, or foam cell/giant cell reaction. In addition, a comparable proportion of responders and nonresponders was seen in both groups.

Many studies have been performed examining neoadjuvant therapy in patients with locally advanced (Stage IIIA) lung cancer (2-8). As a result, neoadjuvant therapy is commonly employed in patients with Stage IIIA lung cancer. Neoadjuvant trials have used chemotherapy or chemotherapy in combination with radiation therapy; however, there has not been a randomized trial that compares these two neoadjuvant strategies. Radiation therapy in addition to preoperative chemotherapy has been associated with a higher incidence of postoperative complications (19-21). We could not find an association between a particular tumor response pattern and type of therapy (chemotherapy or chemoradiation), and responders included patients that received either modality. Given the importance of complete pathologic response and potentially extensive pathologic response in prognosis, the confirmation of this observation is of importance for the design of future neoadjuvant trials.

Several observations with regard to histology can be made. Squamous carcinoma was associated with a higher probability of treatment response than adenocarcinomas, and adenocarcinomas were the histology of the marginal responders (Group Ia). Pretreatment was often recognizable because of the frequent mixture of foam cells and giant cells, in addition to either a predominance of infarct-like coagulative necrosis or fibrosis. The histologic growth pattern of the carcinomas was unchanged by therapy, with the exception of a recognizable increase in nuclear grade in some cases. Of note, no cases showed what might be described as dedifferentiation; that is, a growth pattern associated with a more aggressive tumor type (e.g., large cell carcinoma, small cell carcinoma, or sarcomatoid carcinoma). In nonresponders, it was often not possible to recognize neoadjuvant treatment effects based on tumor histology alone.

In some cases, the residual tumor was largely bronchiolo-alveolar carcinoma pattern, possibly reflecting resistance to therapy in this histologic pattern. Given the possibility that this pattern of growth reflects a form of noninvasive carcinoma, it remains unclear whether a predominance of bronchiolo-alveolar carcinoma pattern in the residual tumor defines a distinct subset of nonresponders in this setting. Conclusions regarding this subset await larger series of patients, possibly series that include earlier stage patients.

Comparison of pathologic tumor regression and preoperative radiologic assessment of size reduction did not show a statistically significant correlation. Also, prediction of pathologic complete response by size reduction was not accurate, and some patients in the pathologic nonresponder group had partial radiologic responses. This finding has been reported elsewhere $(9,12)$. Linking histologic patterns of response revealed that in cases with $\geq 10 \%$ response (Subgroups Ib, IIa, and IIb), degree of fibrosis in fact correlated with size reduction estimates. It is possible that the observed shrinkage seen radiologically is related to retraction associated with fibrosis. This possibility has been raised previously by Seto et al. (22). In their study, the shape of the residual tumor nodule was of im- 
port, and the development of a concave profile was included as a response criterion. They postulated that retraction from fibrosis contributed to this change in shape. Unfortunately, although this correlation may explain some of the observations, extensive fibrosis did not predict minimal or absent residual tumor, so that overall size reduction did not predict absence of significant amounts ( $>10 \%)$ of residual carcinoma. Also, the variation in predicted tumor response was also seen in the marginal response group (Group Ia), a finding that is not explained by the observed differences in extent of fibrosis. It is likely that advances in imaging may assist in resolving this problem. Three-dimensional reconstructions show promise in better elucidating asymmetric growth, and PET scanning may demonstrate residual tumor activity after neoadjuvant therapy (23).

Our survival analysis is limited by a small sample size. Among our Stage IIIA or higher patients, neither survival nor event-free survival duration achieved a level of statistical significance when divided among the two tumor regression groups. When only Stage IIIA patients were examined, there appeared to be a positive trend towards survival, but this did not achieve statistical significance. In a larger study, Junker et al. (12) demonstrated a survival advantage in Stage IIIA responders (complete and extensive response combined).

Given these findings, the recognition of cases in which neoadjuvant therapy has been administered is paramount, and the identification of these cases requires some clinical input. Although many of the changes seen were consistent between cases, finding fibrosis and necrosis associated with giant cell reaction could lead to alternative interpretations of infectious and inflammatory processes, especially when no residual tumor is identified. In such cases, it is imperative that the entire mass is submitted and that therefore, an indication from the treating physician that neoadjuvant therapy has been given is required. This information may become of even greater relevance if neoadjuvant therapy is used in Stage I and II tumors. In such instances, the pathologist may not be able to easily recognize that a cancer operation has been performed and may fail to submit the entire lesion.

In summary, classification of tumor regression in non-small cell lung carcinoma into four subgroups can be performed, generating responder and nonresponder groups. Histologic changes common to these cases are fibrosis, necrosis, and foam cell/ giant cell reaction, as well as increase in residual tumor nuclear grade in some cases. Radiologic assessments do not correlate with prediction of these groups, although they may correlate with extent of fibrosis within the tumor.

\section{REFERENCES}

1. Travis WD, Colby TV, Corrin B, Shimosato Y, Brambilla E. Histological typing of lung and pleural tumors. 3rd ed. Germany: Springer, 1999.

2. Strauss GM, Herndon JE, Sherman DD, Mathisen DJ, Carey RW, Choi NC, et al. Neoadjuvant chemotherapy and radiotherapy followed by surgery in stage IIIA non-small-cell carcinoma of the lung: report of a cancer and leukemia group B phase II study. J Clin Oncol 1992;10:1237-44.

3. Choi NC, Carey RW, Daly W, Mathisen D, Wain J, Wright C, et al. Potential impact on survival of improved tumor downstaging and resection rate by pre-operative twice daily radiation and concurrent chemotherapy in Stage IIIA non small cell cancer. J Clin Oncol 1997;15:712-22.

4. Albain KS, Rusch VW, Crowley JJ, Rice TW, Turrisi AT, Weick $\mathrm{JK}$, et al. Concurrent cisplatin/etoposide plus chest radiotherapy followed by surgery for Stages IIIA and IIIB nonsmall cell lung cancer: mature results of SWOG Phase II study 8805. J Clin Oncol 1995;13:1880-92.

5. Roth JA, Fossella F, Komaki R, Ryan MB, Putnam JB Jr, Lee JS, et al. A randomized trial comparing perioperative chemotherapy and surgery with surgery alone in respectable IIIA non-small cell lung cancer. J Natl Cancer Inst 1994;86:67380.

6. Roth JA, Atkinson EN, Fossella F, Komaki R, Ryan M, Putnam $\mathrm{JB}$, et al. Long term follow-up of patients enrolled in a randomized trial comparing peri-operative chemotherapy and surgery with surgery alone in resectable Stage IIIA nonsmall cell lung cancer. Lung Cancer 1998;21:1-6.

7. Rosell R, Gomez-Codina J, Camps C, Javier Sanchez J, Maestre J, Padilla J, et al. Preresectional chemotherapy in stage IIIA non small cell lung cancer: a 7 year assessment of a randomized clinical trial. Lung Cancer 1999;26:7-14.

8. Pisters KM, Kris MG, Gralla RJ, Zaman MB, Heelan RT, Martini N. Pathologic complete response in advanced non-small-cell lung cancer following preoperative chemotherapy: implications for the design of future non-small-cell lung cancer combined modality trials. J Clin Oncol 1993;11:1757-62.

9. Pisters KM, Ginsberg RJ, Giroux DJ, Putnam JB Jr, Kris MG, Johnson DH, et al. Induction chemotherapy before surgery for early-stage lung cancer: a novel approach. Bimodality lung oncology team. J Thorac Cardiovasc Surg 2000;119:429-39.

10. Depierre A, Milleron B, Moro-Sibilot D, Chevret S, Quoix E, Lebeau B, et al. Preoperative chemotherapy followed by surgery compared with primary surgery in resectable stage I (except T1N0), II and IIIa non-small cell cancer. J Clin Oncol 2002;20:247-53.

11. Junker K, Thomas M, Schulmann K, Klinke F, Bosse U, Müller KM. Tumor regression in non-small-cell lung cancer following neoadjuvant therapy. Histological assessment. Cancer Res Clin Oncol 1997;123:469-77.

12. Junker K, Langner K, Klinke F, Bosse U, Thomas M. Grading of tumor regression in non-small cell lung cancer. Morphol Prognosis Chest 2001;120:1584-91.

13. Mountain CF. The International System for Staging Lung Cancer. Semin Surg Oncol 2000;18:106-15.

14. Picci P, Bacci G, Campanacci M, Gasparini M, Pilotti S, Cerasoli $\mathrm{S}$, et al. Histologic evaluation of necrosis in osteosarcoma induced by chemotherapy. Cancer 1985;56:1515-21.

15. Rosen G, Caparros B, Huvos A, Kosloff C, Nirenberg A, Cacavio A, et al. Preoperative chemotherapy for osteogenic sarcoma: selection of postoperative adjuvant chemotherapy based on the response of the primary tumor to preoperative chemotherapy. Cancer 1982;49:1221-30.

16. Sulfaro S, Frustaci S, Volpe R, Barzan L, Comoretto R, Monfardini S, et al. A pathologic assessment of tumor residue and stromal changes after intraarterial chemotherapy for head and neck carcinomas. Cancer 1989;64:994-1001. 
17. Mandard AM, Dalibard F, Mandard JC, Marnay J, HenryAmar M, Petiot JF, et al. Pathologic assessment of tumor regression after preoperative chemotherapy of esophageal carcinoma. Cancer 1994;73:2680-6.

18. Fushimi H, Kikui M, Morino H, Yamamoto S, Tateishi R, Wada A, et al. Histologic changes in small cell lung carcinoma after treatment. Cancer 1996;77:278-83.

19. Deutsch M, Crawford J, Leopold K, Wolfe W, Foster W, Herndon J, et al. Phase II study of neoadjuvant chemotherapy and radiation therapy with thoracotomy in the treatment of clinically staged IIIA non-small cell lung cancer. Cancer 1994;74:1243-52.

20. Fowler WC, Langer CJ, Curran WJ Jr, Keller SM. Postoperative complications after combined neoadjuvant treatment of lung cancer. Ann Thorac Surg 1993;55:986-9.
21. Bonomi P, Faber LP, Warren W, Lincoln S, LaFollette S, Sharma M, et al. Postoperative bronchopulmonary complications in stage III lung cancer patients treated with preoperative paclitaxel-containing chemotherapy and concurrent radiation. Semin Oncol 1997;24:S12-123-9.

22. Seto M, Kuriyama K, Kasugai T, Kido S, Sawai Y, Kuroda T, et al. Comparison of computed tomography and pathologic examination for evaluation of response of primary lung cancer to neoadjuvant therapy. J Thorac Imaging 1999;14:69-73.

23. Choi NC, Fischman AJ, Niemierko A, Ryu JS, Lynch T, Wain $\mathrm{J}$, et al. Dose-response relationship between probability of pathologic tumor control and glucose metabolic rate measured with FDG PET after preoperative chemoradiotherapy in locally advanced non-small-cell lung cancer. Int J Radiat Oncol Biol Phys 2002;54:1024-35. 